\title{
Rhodium complexes with $N$-phenyl anthranilic acid ligands as catalysts for hydrogenation
}

\author{
T.G. Ros, M.K. van der Lee, A.J. van Dillen, J.W. Geus, D.C. Koningsberger* \\ Department of Inorganic Chemistry and Catalysis, Debye Institute, Utrecht University, P.O. Box 80083, 3508 TB Utrecht, The Netherlands
}

Received 24 October 2001; received in revised form 12 February 2002; accepted 16 February 2002

\begin{abstract}
The complexes of rhodium with $N$-phenyl anthranilic acid and anthranilic acid were synthesised and their structure was investigated with infrared spectroscopy. It appears that rhodium interacts with $N$-phenyl anthranilic acid via the carboxyl and the amine groups. With anthranilic acid, the bond with the amine could not be unambiguously established. X-ray diffraction and elemental analysis data suggest that the complexes are not pure and that the presence of $\mathrm{Rh}(0)$ is likely. Catalytic hydrogenation results and mercury poisoning experiments confirm the presence of $\mathrm{Rh}(0)$. ( $) 2002$ Elsevier Science B.V. All rights reserved.
\end{abstract}

Keywords: Catalytic hydrogenation; Infrared spectroscopy; Mercury poisoning; $N$-phenyl anthranilic acid; Rhodium complexes

\section{Introduction}

Chekrii and co-workers [1-4] first reported on the rhodium complex with $N$-phenyl anthranilic acid (PAA) in the late 1960s. The structure of PAA is shown in Fig. 1. The $N, N$-dimethylformamide (DMF)-soluble $\mathrm{Rh}(\mathrm{I})$ complex with PAA was claimed to be a remarkably active hydrogenation catalyst. Benzene and polyaromatic compounds were hydrogenated rapidly at room temperature and 1 atm hydrogen pressure [3]. The complex was synthesised by heating equimolar amounts of rhodium chloride and PAA in ethanol followed by treatment of the reaction mixture with sodium borohydride [4]. Various analytical data suggested that the $N$-phenyl anthranilic acid (RhPAA) complex was a dimer [1]. The mono-substituted ring of the $\mathrm{N}$-phenyl anthranilate ion forms a half-sandwich

\footnotetext{
* Corresponding author. Tel.: +31-30-2537400; fax: +31-30-2511027.

E-mail address: d.c.koningsberger@chem.uu.nl (D.C. Koningsberger).
}

structure through $\pi$ bonds with one of the rhodium atoms, while the ionised carboxyl group co-ordinates to the second rhodium atom.

The RhPAA catalyst has not received much attention, although it was the first to be used in the hydrogenation of aromatic compounds. It has been doubted severely whether the catalyst is really homogeneous [5-7]. The proposed structure has also been disputed. The suggested interaction of rhodium with the phenyl ring was based on very small changes in the frequencies of the infrared (IR) bands of the $\mathrm{C}-\mathrm{H}$ vibrations. In the absence of other data, such slight band shifts are a questionable proof of metal-ring bonds [7,8]. Moreover, the IR spectrum published differed from the tabulated band shifts [2]. Finally, the elemental analysis data were not in agreement with the proposed structure [7].

In view of the above uncertainties concerning the structure and the homogeneity of the RhPAA complex, Borowski [7] studied the system again. The synthesis procedure of Chekrii and co-workers was modified in order to prevent reduction of unreacted rhodium 


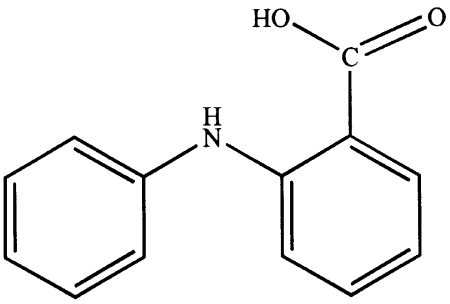

PAA

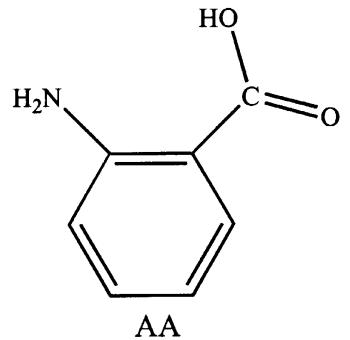

Fig. 1. Structure of PAA and AA.

chloride to metallic rhodium by sodium borohydride. Borowski and co-workers also pointed out that the ethanol used in the synthesis is already capable of reducing the rhodium to $\mathrm{Rh}(0)$. Based on an IR study of the complex, it was established that the Rh was co-ordinated to the ionised carboxyl group in a bridging mode (bridging between two Rh atoms, thus suggesting a dimer structure). No interaction of Rh with the phenyl or the amine group was found. However, a definite structure of the RhPAA complex could not be given. It was postulated that the complex consisted of large molecules linked by carboxylate and/or chloride ions. In other publications [9-11], the use of the rhodium complex with anthranilic acid (AA), presumably obtained according to the same synthesis procedure, was reported. The structure of AA is given in Fig. 1. Borowski and co-workers claimed hydrogenation activities of RhPAA to be an order of magnitude higher than those found by Chekrii and co-workers. Contrary to the first report, the later publications stated that in both complexes the rhodium binds to the ligand by co-ordination to both the carboxylate and the amine group. Unfortunately, no experimental evidence was given.

The current interest in the RhPAA and rhodium anthranilic acid (RhAA) complexes was initiated by publications by Holy $[6,12,13]$, who immobilised these catalysts onto polystyrene. Upon immobilisation of RhPAA, the catalyst did not display any activity. When AA instead of PAA was used, a highly active immobilised $\mathrm{Rh}(\mathrm{I})$ catalyst was obtained. The immobilised complex also showed hydrogenation activity with $\mathrm{Ni}$ as the active metal [14]. The AA complex is an example of an immobilised complex that does not have a homogeneous analogue. However, polystyrene is not a very suitable support material for application of the catalyst in an industrial environment. In our laboratory, we intend to immobilise the RhAA complex onto carbon nanofibres (CNFs), which are mechanically strong and have a mesoporous macrostructure. In order to be able to execute the immobilisation, more knowledge is needed about the homogeneous RhPAA and RhAA systems. More insight is desired about the factors that influence the synthesis procedure and more information should be obtained about the interaction of $\mathrm{Rh}$ with the different functional groups of PAA and AA. Furthermore, the homogeneity of the complexes has to be thoroughly checked.

In this study, we have performed the synthesis of RhPAA and RhAA based on the procedure used by Borowski [7]. The interaction of the ligands with $\mathrm{Rh}$ was studied with IR spectroscopy. Emphasis was put on investigating whether the complexes are genuinely homogeneous. One of the techniques used was mercury poisoning, a well-described and accepted technique in literature [15-19]. Mercury selectively poisons the catalytic activity of metal particles and not that of homogeneous complexes. Therefore, addition of mercury can establish whether the catalytic activity originates from a metal complex or from small metal particles. It will be shown that Rh interacts with PAA via the carboxyl and the amine group, whereas with AA the bond with the amine could not be established definitively. The systems turned out to be impure and the presence of $\mathrm{Rh}(0)$ is very likely.

\section{Experimental}

\subsection{Synthesis of RhPAA}

The synthesis procedure of RhPAA was derived from the preparation method used by Borowski [7]. 
Every synthesis step was carried out under a nitrogen atmosphere, using standard Schlenk techniques. Solvents (demineralised water and pro-analyse ethanol from Merck) were degassed by vacuum suction prior to use. AA $(98+\%)$, PAA (98\%) and $\mathrm{RhCl}_{3} \cdot 2 \mathrm{H}_{2} \mathrm{O}$ (99.9\%) were purchased from Acros. $\mathrm{NaBH}_{4}(99 \%)$ was obtained from Alfa.

Equimolar amounts of $\mathrm{RhCl}_{3} \cdot 2 \mathrm{H}_{2} \mathrm{O} \quad(1.00 \mathrm{~g}$, $4 \mathrm{mmol})$ and PAA $(0.85 \mathrm{~g}, 4 \mathrm{mmol})$ in $50 \mathrm{ml}$ waterethanol (2:1) were refluxed for $1 \mathrm{~h}$. Upon cooling, the dark greenish-black precipitate was separated from the light reddish-pink solution by centrifugation and decantation, washed with water-ethanol (2:1), and dried under vacuum (yield $1.1 \mathrm{~g}$ ). The RhPAA complex was reduced with sodium borohydride by re-suspension in $50 \mathrm{ml}$ water-ethanol $(2: 1)$ at $0{ }^{\circ} \mathrm{C}$ and slow addition of $\mathrm{NaBH}_{4}(0.4 \mathrm{~g}, 0.01 \mathrm{~mol})$ in $4 \mathrm{ml}$ water $\left(0^{\circ} \mathrm{C}\right)$ under vigorous stirring, whereupon the colour of the suspension darkened. The reduced complex was separated by centrifugation and decantation, washed with water-ethanol $(2: 1)$, and dried under vacuum (yield $0.7 \mathrm{~g}$ ).

Equimolar amounts of $\mathrm{RhCl}_{3} \cdot 2 \mathrm{H}_{2} \mathrm{O} \quad(1.00 \mathrm{~g}$, $4 \mathrm{mmol})$ and AA $(0.52 \mathrm{~g}, 4 \mathrm{mmol})$ in $50 \mathrm{ml}$ waterethanol (2:1) were refluxed for 1 and $20 \mathrm{~h}$. Upon cooling, the precipitate was separated by centrifugation and decantation, washed with water-ethanol (2:1), and dried under vacuum. Refluxing for 1 and $20 \mathrm{~h}$ resulted in yields of 0.4 and $0.6 \mathrm{~g}$, respectively. Upon refluxing for $1 \mathrm{~h}$, the colour of the precipitate was greenishbrown, whereas after reaction for $20 \mathrm{~h}$ the colour had changed to black. In both cases, the colour of the solution was yellowish-orange. The RhAA complex was reduced with sodium borohydride by re-suspension in $50 \mathrm{ml}$ water-ethanol $(2: 1)$ at $0{ }^{\circ} \mathrm{C}$ and slow addition of $\mathrm{NaBH}_{4}(0.4 \mathrm{~g}, 0.01 \mathrm{~mol})$ in $4 \mathrm{ml}$ water $\left(0^{\circ} \mathrm{C}\right)$ under vigorous stirring. The reduced complex was separated by centrifugation and decantation, washed with water-ethanol (2:1), and dried under vacuum (yield $0.5 \mathrm{~g}$ for the $20 \mathrm{~h}$ synthesis). The colour of the RhAA complex that was refluxed for only $1 \mathrm{~h}$ changed to black upon reduction with $\mathrm{NaBH}_{4}$.

For reasons of comparison, metallic Rh on CNFs $(\mathrm{Rh} / \mathrm{CNFs}, 1 \mathrm{wt} . \%)$ was prepared by heating under reflux $3.0 \mathrm{~g}$ of fishbone CNFs in a solution of $73 \mathrm{mg}$ $\mathrm{RhCl}_{3} \cdot 2 \mathrm{H}_{2} \mathrm{O}$ in ethanol for $2 \mathrm{~h}$. The catalyst was filtered from the colourless solution, washed with ethanol and air-dried.

\subsection{The characterisation of RhPAA}

Elemental analyses were performed by Dornis und Kolbe, Mikroanalytisches Laboratorium (Mülheim, Germany).

Powder XRD measurements were carried out using an ENRAF-NONIUS X-ray diffraction (XRD) system equipped with a curved position-sensitive INEL detector operating up to a width of $120^{\circ} 2 \theta$. The applied radiation was Co $\mathrm{K} \alpha(\lambda=1.78897 \AA)$. Powdered samples and dispersions in degassed silicon grease (under $\mathrm{N}_{2}$ ) were prepared.

Transmission IR data were recorded on a Perkin Elmer 2000 spectrometer equipped with an air dryer for removal of water vapour and carbon dioxide. One hundred scans were co-added at a resolution of $4 \mathrm{~cm}^{-1}$ and a boxcar apodisation. Samples were prepared by dispersion of the complexes in degassed paraffin oil (spectroscopy grade) under $\mathrm{N}_{2}$ (measured between $\mathrm{NaCl}$ windows) as well as by dispersion in pre-dried $\mathrm{KBr}$. Tablets were pressed at $4 \mathrm{t} / \mathrm{cm}^{2}$ in vacuum for $2 \mathrm{~min}$. Sample concentrations were about $1 \%(\mathrm{~mol} / \mathrm{mol})$ in $\mathrm{KBr}$.

First, spectra of the complexes were measured in paraffin oil before and after exposure to air for several days. No difference in the spectra was found and therefore all other spectra were obtained with $\mathrm{KBr}$ disks.

\subsection{Catalytic test experiments}

\subsubsection{General}

The catalytic activity of the Rh complexes and the $\mathrm{Rh} / \mathrm{CNFs}$ catalyst for the hydrogenation of cyclohexene was measured in a semi-batch slurry reactor, operated at a constant pressure of $1200 \mathrm{mbar}^{\mathrm{H}_{2}}$. The thermostated, double-walled reaction vessel was equipped with vertical baffles and a gas-tight stirrer with a hollow shaft and blades for gas recirculation. The stirrer was operated at $2000 \mathrm{rpm}$. Solvent (DMF, Merck p.a.) and reactant (cyclohexene, Acros 99\%) were degassed by vacuum suction prior to use. During reaction, the hydrogen consumption was automatically monitored by a mass flow meter. It was assured that, under the conditions used, the rate of dissolution of $\mathrm{H}_{2}$ in DMF was higher than the maximum measurable rate of $\mathrm{H}_{2}$-uptake.

In a typical experiment, the reaction vessel was evacuated and filled with $\mathrm{N}_{2}$, where after a solution 
of the complex in DMF (14-18 mg in $100 \mathrm{ml}$ DMF, $500 \mathrm{mg}$ for $\mathrm{Rh} / \mathrm{CNFs}$ ) was transferred into the vessel under a stream of nitrogen. Next, the reactor was thermostated at $25^{\circ} \mathrm{C}$, evacuated, filled with $\mathrm{H}_{2}$ and pressurised. The reaction was started by injection of $0.5-5 \mathrm{ml}$ cyclohexene with a syringe and was allowed to proceed until total hydrogenation of the cyclohexene.

\subsubsection{Mercury poisoning experiments}

A first catalytic run was executed with $1 \mathrm{ml}$ cyclohexene to check the activity of the catalyst under investigation. After total hydrogenation of the added reactant, another dose of $5 \mathrm{ml}$ of cyclohexene was injected. The hydrogenation of the second dose of cyclohexene was stopped after $3 \mathrm{~min}$ by turning off the stirrer, removal of the $\mathrm{H}_{2}$ by evacuation and addition of $\mathrm{N}_{2}$. Next, the temperature was raised to $50^{\circ} \mathrm{C}$ and mercury (mole ratio of $\mathrm{Hg}$-to-Rh 300:1) was injected with a syringe. After stirring for $3 \mathrm{~h}$ at this temperature, the reaction mixture was cooled to $25^{\circ} \mathrm{C}, \mathrm{H}_{2}$ was admitted and the catalytic run was continued.

\section{Results}

\subsection{Characterisation}

\subsubsection{General}

The very dark-brown to black complexes turned out to be only sparingly soluble in organic solvents. The RhAA complex is soluble in DMF up to about $0.1 \mathrm{~g} / \mathrm{l}$, while the RhPAA complex is soluble in DMF ( $>1 \mathrm{~g} / \mathrm{l}), 3$-pentanon $(\sim 0.5 \mathrm{~g} / \mathrm{l})$, pyridine $(\sim 0.6 \mathrm{~g} / \mathrm{l})$ and tetrahydrofuran $(\sim 0.4 \mathrm{~g} / \mathrm{l})$. Due to the low solubility of the complexes, we were not able to obtain NMR spectra on a Varian Inova $300 \mathrm{MHz}$ spectrometer in DMF/benzene-d6. Furthermore, attempts to crystallise the complexes from DMF solutions (in an ethanol vapour environment) failed. The attempts led only to the formation of metallic rhodium.

\subsubsection{Elemental analysis}

Table 1 represents the results of the elemental analysis of RhPAA and RhAA. The relative content of carbon, hydrogen, nitrogen and chlorine was determined.
Table 1

Results of the elemental analysis of rhodium RhPAA and RhAA

\begin{tabular}{lllll}
\hline & $\mathrm{C}(\%)$ & $\mathrm{H}(\%)$ & $\mathrm{N}(\%)$ & $\mathrm{Cl}(\%)$ \\
\hline RhPAA & 40.69 & 3.70 & 2.97 & 0.65 \\
RhAA & 26.08 & 3.05 & 3.23 & 4.63 \\
\hline
\end{tabular}

\subsubsection{X-ray diffraction}

The XRD patterns of RhPAA and RhAA measured in air show typical rhodium metal reflections at $d$-values of 2.196, 1.902, 1.345 and $1.147 \AA$ (diffraction patterns not shown). The width at half height of these peaks was used to calculate the average rhodium particle diameter using the Scherrer equation [20]. Average particle sizes of 5-6 nm were found. Besides the Rh peaks, the X-ray pattern of RhAA did not show any other reflections. The diffractogram of RhPAA, however, exhibited some sharp peaks at low $2 \theta$ values $\left(<20^{\circ} 2 \theta\right)$.

In order to establish whether metallic rhodium is already present in the complexes after synthesis or whether the metal is formed after contact with air, the complexes were also measured in silicon grease. Fig. 2 displays the results for RhPAA. As shown, the diffractogram of silicon grease alone already shows some peaks between $9^{\circ}$ and $32^{\circ} 2 \theta$. Due to the dispersion in silicon grease, the intensity of all rhodium peaks decreased considerably and only the most intense Rh $d\left(\begin{array}{lll}1 & 1 & 1\end{array}\right)$ reflection at $48.1^{\circ} 2 \theta$ could be monitored. After synthesis, the RhPAA complex does not show any metallic rhodium. However, after reaction or after contact with air for one night, some metallic rhodium can be detected. The measurement after reaction was repeated (i.e., another catalytic measurement was performed with the RhPAA complex and the XRD pattern of the complex after reaction was measured.) and in this case no $\mathrm{Rh}(0)$ was detected. The RhAA complex also showed no rhodium after synthesis, whereas after one night in air the metal was evident in the X-ray pattern.

The average particle size of the $1 \mathrm{wt} . \% \mathrm{Rh} / \mathrm{CNFs}$ catalyst was also determined with XRD. The average diameter found was $11 \mathrm{~nm}$.

\subsubsection{IR spectroscopy}

Fig. 3 shows the IR spectra of PAA and RhPAA. The assignments of relevant bands are summarised in 


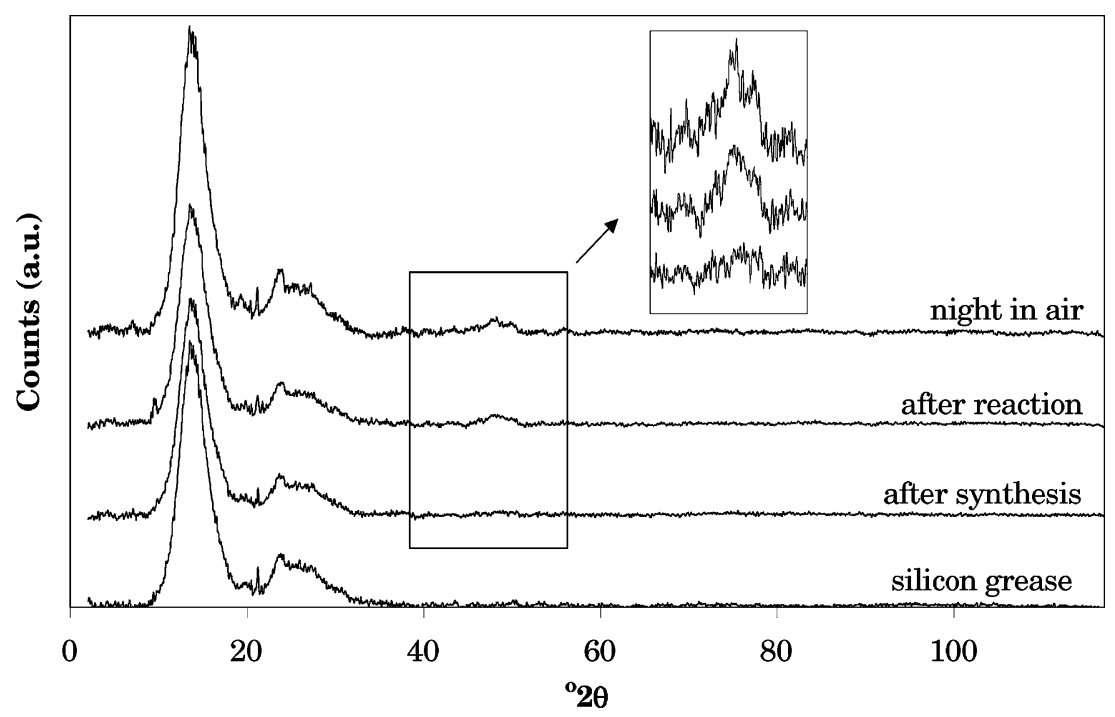

Fig. 2. XRD patterns of RhPAA in silicon grease after several treatments.

Table 2. Besides aromatic ring and other vibrations, two significant vibrations at 1657 and $1261 \mathrm{~cm}^{-1}$ occur in the spectrum of PAA. The band at $1657 \mathrm{~cm}^{-1}$ is attributed to the $\mathrm{C}=\mathrm{O}$ stretching vibration of the carboxyl group [2,21] and that at $1261 \mathrm{~cm}^{-1}$ can be ascribed to the $\mathrm{C}-\mathrm{N}$ stretching vibration [21]. Upon complexation with rhodium, the $\mathrm{C}=\mathrm{O}$ stretching vibration disappears and two peaks at 1612 and $1386 \mathrm{~cm}^{-1}$ emerge. The two peaks are assigned to the antisymmetric and the symmetric stretching vibration of the ionised carboxylate group, respectively $[2,21]$. Furthermore, the $\mathrm{C}-\mathrm{N}$ stretching vibration at $1261 \mathrm{~cm}^{-1}$ shifts 20 to $1281 \mathrm{~cm}^{-1}$. The new broad band at $523 \mathrm{~cm}^{-1}$ originates from the Rh-N stretching vibration [22]. Finally, the $\mathrm{N}-\mathrm{H}$ stretching vibration at $3334 \mathrm{~cm}^{-1}$ is broadened and slightly shifted to $3343 \mathrm{~cm}^{-1}$ (band not shown in Fig. 3). When the complex had been "aged" for several months under

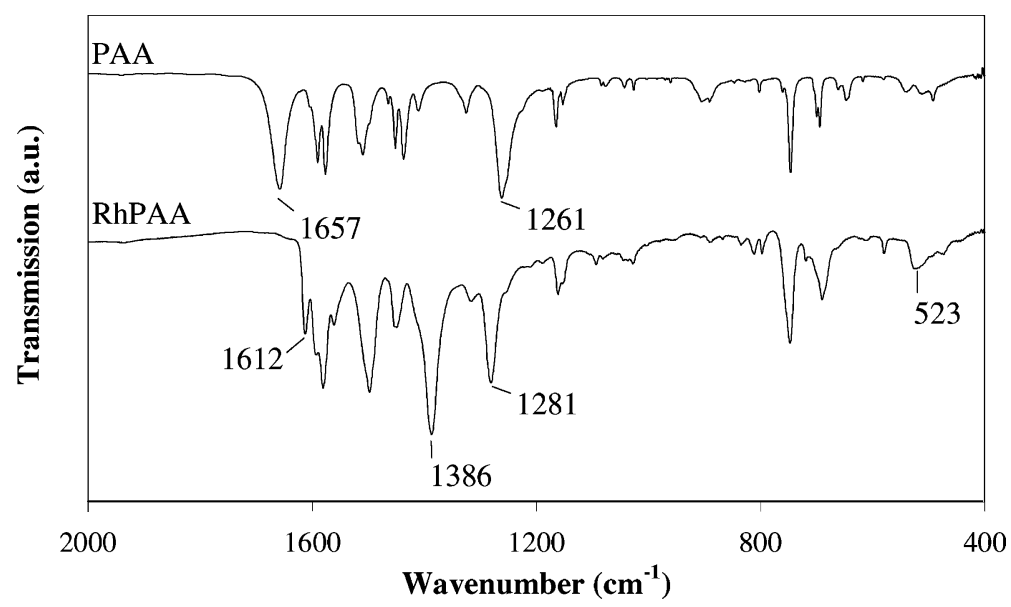

Fig. 3. IR spectra of PAA and RhPAA. 
Table 2

Assignments of important bands in the spectra of PAA, RhPAA, AA and RhAA

\begin{tabular}{lll}
\hline $\begin{array}{l}\text { Wavenumber } \\
\left(\mathrm{cm}^{-1}\right)\end{array}$ & Assignment & Reference \\
\hline $1664-1657$ & $\mathrm{C}=\mathrm{O}$ stretching & {$[2,21]$} \\
$1612-1610$ & Antisymmetric $\mathrm{COO}^{-}$stretching & {$[2,21]$} \\
1531 & $\mathrm{NH}_{2}$ bending & {$[21]$} \\
$1392-1386$ & $\mathrm{Symmetric} \mathrm{COO}^{-}$stretching & {$[2,21]$} \\
$1281-1261$ & $\mathrm{C}-\mathrm{N}$ stretching & {$[21]$} \\
523 & Rh-N stretching & {$[22]$} \\
\hline
\end{tabular}

$\mathrm{N}_{2}$, a weak band at $1643 \mathrm{~cm}^{-1}$ was present. This absorption probably originates from the $\mathrm{C}=\mathrm{O}$ stretching vibration of the carboxyl group. The results described above were reproduced with repeated syntheses of the complex.

Fig. 4 represents the IR spectra of AA and RhAA complex. As the spectrum of AA is very rich in the range displayed, the assignment of relevant bands is more difficult than with PAA. However, the $\mathrm{C}=\mathrm{O}$ stretching vibration at $1664 \mathrm{~cm}^{-1}$ can easily be distinguished. Similar to PAA the carboxyl group ionises upon complexation, resulting in the antisymmetric and symmetric $\mathrm{COO}^{-}$stretching vibrations at 1610 and $1392 \mathrm{~cm}^{-1}$, respectively. Additionally, a band appears at $1531 \mathrm{~cm}^{-1}$, which can be ascribed to a $\mathrm{NH}_{2}$ bending vibration. This peak could be the shifted absorption that is visible as a shoulder at $1554 \mathrm{~cm}^{-1}$ in the spectrum of AA. In contrast to the spectrum of RhPAA, that of RhAA displays only few bands and hardly resembles the spectrum of AA. Therefore, no statements can be made about the presence or absence of $\mathrm{Rh}-\mathrm{N}$ and $\mathrm{C}-\mathrm{N}$ vibrations. Lastly, the two sharp $\mathrm{N}-\mathrm{H}$ stretching vibrations at 3324 and $3237 \mathrm{~cm}^{-1}$ are replaced by weak, broad bands at 3434 and $3232 \mathrm{~cm}^{-1}$, which could well be due to adsorbed water or lattice water [23] (bands not shown in Fig. 4). Spectra of the RhAA complexes that were obtained by refluxing for either 1 or $20 \mathrm{~h}$ were identical, as were the spectra of the complex before and after reduction with $\mathrm{NaBH}_{4}$ ( $1 \mathrm{~h}$ reflux).

\subsection{Catalytic activity}

\subsubsection{General}

In Fig. 5, the $\mathrm{H}_{2}$-flow patterns and the $\mathrm{H}_{2}$-uptake curves of RhPAA and RhAA for the hydrogenation of $1 \mathrm{ml}$ cyclohexene are shown. The $\mathrm{H}_{2}$-flow pattern was integrated to get the $\mathrm{H}_{2}$-uptake curve, which was used to calculate initial activities (i.e., the activity at $t=0 \mathrm{~min}$ ). Initial activities of 7 and $6 \mathrm{~mol} \mathrm{H}_{2} / \mathrm{g}_{\text {cat }} \mathrm{h}$ were found for RhPAA and RhAA, respectively. The values for the initial activities were difficult to reproduce. With RhPAA deviations of up to $20 \%$ were found. Upon storage under nitrogen, the activity of the complex showed a strong deactivation in time of up to $75 \%$. With RhAA this deactivation was also observed. Not much difference in activity before and after reduction was found with the RhAA complex. With the

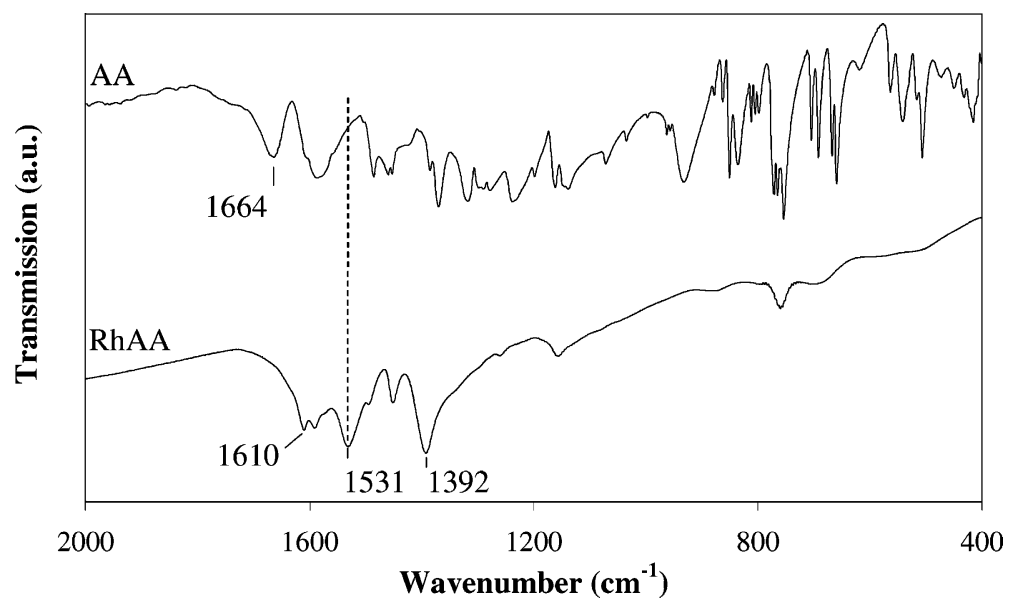

Fig. 4. IR spectra of AA and RhAA. 

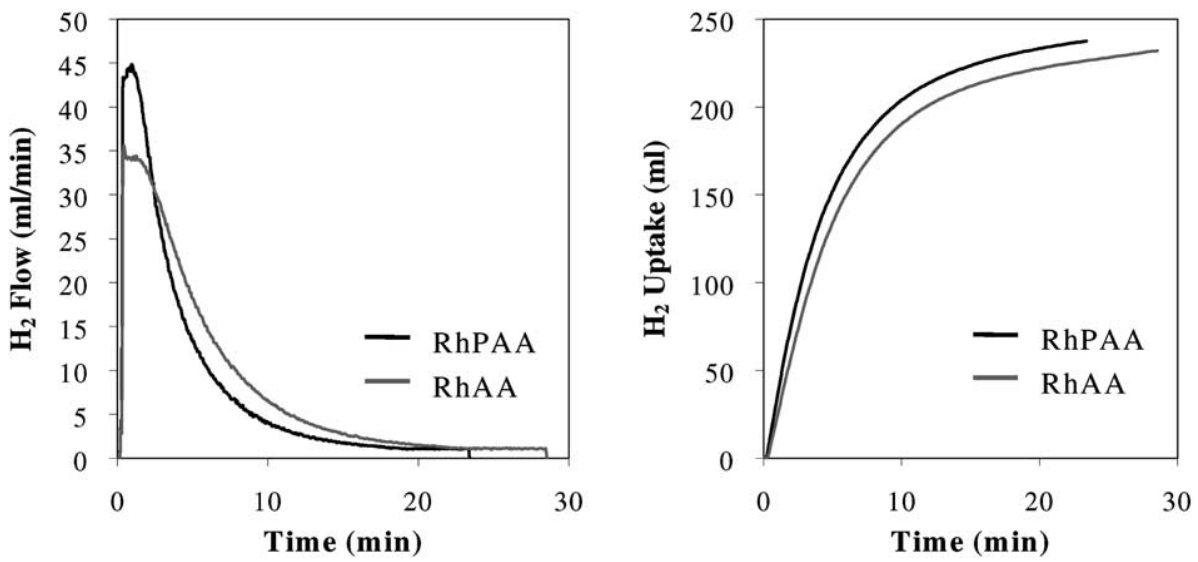

Fig. 5. $\mathrm{H}_{2}$-flow patterns (a) and $\mathrm{H}_{2}$-uptake (b) curves of RhPAA (18.3 mg) and RhAA (14.4 mg) for the hydrogenation of $1 \mathrm{ml}$ cyclohexene.

$1 \mathrm{wt} . \% \mathrm{Rh} / \mathrm{CNFs}$ catalyst, the initial activity amounted only to $0.03 \mathrm{~mol} \mathrm{H}_{2} / \mathrm{g}_{\text {cat }} \mathrm{h}$.

The order with respect to cyclohexene for the RhPAA, RhAA and $\mathrm{Rh} / \mathrm{CNF}$ s catalysts was determined using the power rate law [24]. To this end, the logarithm of the initial activity was plotted against the logarithm of the initial cyclohexene concentration. Fig. 6 displays the results. For RhAA these measurements were carried out with the deactivated complex (i.e., the complex that had been stored under $\mathrm{N}_{2}$ for several weeks). The orders with respect to cyclohexene found are 0.8 for RhPAA, 0.6 for RhAA and 0.7 for $\mathrm{Rh} / \mathrm{CNFs}$.

\subsubsection{Mercury poisoning}

All catalytic runs concerning the mercury poisoning experiments were carried out at $25^{\circ} \mathrm{C}$. The first run with $1 \mathrm{ml}$ cyclohexene was used to determine the initial activity of the catalyst under investigation. The catalytic run with the second dose of cyclohexene (which was interrupted for mercury poisoning) consisted of $5 \mathrm{ml}$ of cyclohexene in order to have enough cyclohexene in the system after mercury poisoning. The initial activities determined after mercury poisoning were thus determined from the continued run with the second dose of $5 \mathrm{ml}$ cyclohexene. Therefore, the results are qualitative.

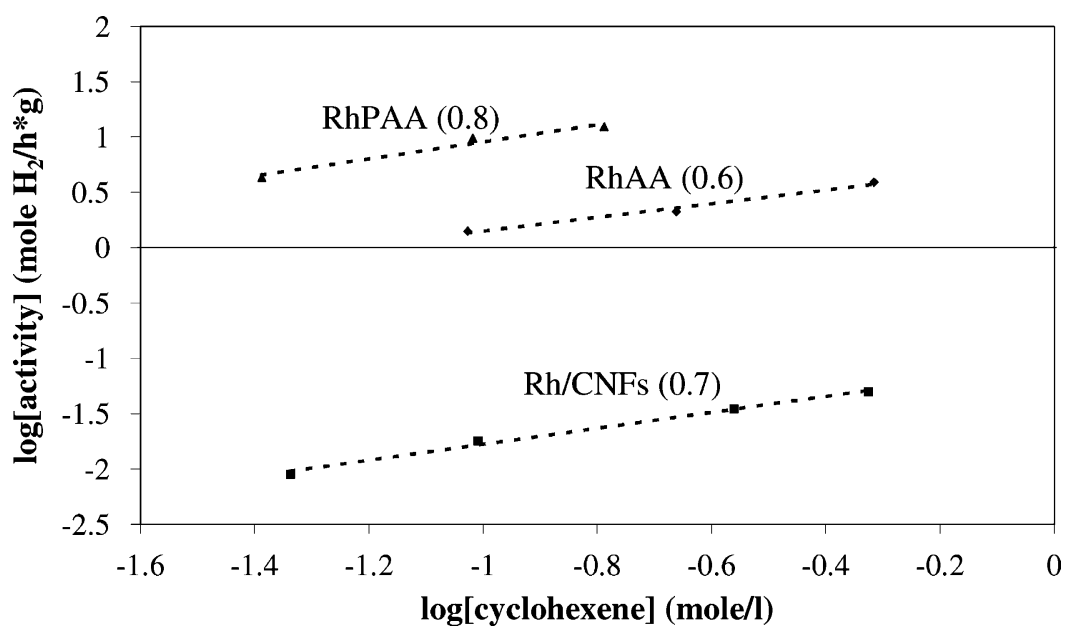

Fig. 6. Determination of the order in cyclohexene for RhPAA, RhAA and Rh/CNFs. 
Table 3

Initial activities of 1 wt.\% Rh/CNFs, RhPAA and RhAA before and after stirring at $50{ }^{\circ} \mathrm{C}$ in DMF (blank) and mercury poisoning at $50^{\circ} \mathrm{C}$ in $\mathrm{DMF}(\mathrm{Hg})$

\begin{tabular}{lll}
\hline Catalyst & \multicolumn{2}{l}{ Initial activity $\left(\mathrm{mol} \mathrm{H}_{2} / \mathrm{g}_{\text {cat }} \mathrm{h}\right)$} \\
\cline { 2 - 3 } & Before treatment & After treatment \\
\hline $\begin{array}{ll}\text { 1 wt.\% Rh/CNFs } \\
\text { Hg }\end{array}$ & 0.03 & 0 \\
RhPAA & & \\
$\quad$ Blank & 9 & 0.2 \\
Hg & 8 & 0 \\
RhAA & & 14 \\
Blank & 4 & 0 \\
Hg & 4 & \\
\hline
\end{tabular}

The concept of poisoning the catalytic activity of $\mathrm{Rh}(0)$ with $\mathrm{Hg}$ was first tested in ethanol with the $1 \mathrm{wt} . \% \mathrm{Rh} / \mathrm{CNF}$ catalyst. The activity of this catalyst was totally blocked after stirring with $\mathrm{Hg}$ in ethanol at $25^{\circ} \mathrm{C}$ for several hours. However, when the experiment with the $\mathrm{Rh} / \mathrm{CNF}$ catalyst was executed in DMF, the activity was not affected. In other words, the run with the second dose of $5 \mathrm{ml}$ cyclohexene was nicely continued after mercury poisoning at the same level as before poisoning. In DMF the reaction mixture had to be stirred at $50{ }^{\circ} \mathrm{C}$ with mercury for several hours before all activity was blocked (see Table 3 ).

Table 3 also presents the results of mercury poisoning of the rhodium complexes at $50^{\circ} \mathrm{C}$ for several hours. In order to investigate the effect of the thermal treatment without the presence of mercury on the activity of the rhodium complexes, the complexes were stirred at $50{ }^{\circ} \mathrm{C}$ for several hours without any mercury added (denoted as "blank"). The initial activities measured before treatment were determined from the first run of cyclohexene, while the initial activities after treatment were calculated from the continued second run. After stirring in DMF at $50{ }^{\circ} \mathrm{C}$ for $3 \mathrm{~h}$ the initial activity of the RhPAA complex had decreased strongly from 9 to $0.2 \mathrm{~mol} \mathrm{H}_{2} / \mathrm{g}_{\text {cat }} \mathrm{h}$. When $\mathrm{Hg}$ was added the initial activity of $8 \mathrm{~mol} \mathrm{H}_{2} / \mathrm{g}_{\text {cat }} \mathrm{h}$ was totally lost. The differences in initial activity before treatment with the RhPAA catalyst reflect the bad reproducibility in catalytic activity. With RhAA the blank experiment resulted in an increase in the initial activity from 4 to $14 \mathrm{~mol} \mathrm{H}_{2} / \mathrm{g}_{\text {cat }} \mathrm{h}$. This activity was subsequently totally blocked by poisoning with $\mathrm{Hg}$. The experiments with RhAA were performed with the complex stored under $\mathrm{N}_{2}$ for several weeks.

The data of Table 3 indicate that the activities of the complexes are much more elevated than that of the $\mathrm{Rh} / \mathrm{CNFs}$ catalyst. This is due to the fact that only $1 \mathrm{wt} . \% \mathrm{Rh}$ was deposited on the CNFs. Accordingly, the initial activity of the catalyst is much lower per gram catalyst than that of the complexes.

\section{Discussion}

\subsection{Elemental analysis}

The results of the elemental analysis point out that the systems under investigation are not simple ones. The data cannot be fitted with the assumption that PAA is co-ordinated to $\mathrm{Rh}$ in a 1:1 ratio and that water or ethanol is also present in the co-ordination sphere. The amounts of chlorine found are too small for reasonable fits with one chlorine atom and, therefore, indicate that the material is polluted. For RhAA the amount of chlorine could also point to the presence of a bridging chloride ion. Reasonable fits of the data may be obtained by assuming that about half of the rhodium is present as $\mathrm{Rh}(0)$ and that PAA and ethanol are co-ordinated to the other half. The rhodium content would then be about $40 \%$ for RhPAA and about $50 \%$ for RhAA. The elemental analysis data differ from the values reported by Borowski [7] for RhPAA (C: 40.15\%; H: 4.1\%; N: 4.9\%; Cl: 3.0\%; Rh: 24.4\%).

\subsection{X-ray diffraction}

The XRD data clearly show that after contact with air small rhodium particles are present in RhPAA. The results obtained with the complex in silicon grease seem to suggest that indeed air induces the formation of metal particles and that in the complexes that are well kept under $\mathrm{N}_{2}$ no $\mathrm{Rh}(0)$ is present. The formation of $\mathrm{Rh}(0)$ might be explained by the assumption that in contact with air by the catalytic action of Rh the PAA is oxidised and that the oxidised ligand subsequently reduces the $\mathrm{Rh}$ [25-29]. The oxidation of the amine group to a nitro group is most likely. The IR absorptions of the nitro group could be obscured by other vibrations occurring at that frequency [21]. However, 
these findings must be interpreted with care. Metal particles smaller than about $2 \mathrm{~nm}$ are not detected by XRD. Moreover, due to the low concentration of the complex in grease it is well possible that not all of the rhodium metal present is detected. Because the same trends were observed with RhPAA and RhAA, it is probable that the amount of $\mathrm{Rh}(0)$ increases after exposure to air. Nevertheless, with IR no difference was found in the spectra after exposure to air. For instance, no $\mathrm{C}=\mathrm{O}$ vibration at $1643 \mathrm{~cm}^{-1}$ was observed. Hence, the increase in $\operatorname{Rh}(0)$ content should not be very significant. Taking into account the synthesis procedure, it is likely that the complexes have been already polluted with rhodium metal before exposure to air, because ethanol is capable of reducing rhodium chloride at elevated temperatures. This is exemplified by the synthesis of $\mathrm{Rh} / \mathrm{CNFs}$ by refluxing $\mathrm{RhCl}_{3} \cdot 2 \mathrm{H}_{2} \mathrm{O}$ in ethanol. Furthermore, the strong reductant $\mathrm{NaBH}_{4}$ can also easily reduce $\mathrm{Rh}(\mathrm{III})$ to $\mathrm{Rh}(0)$.

\subsection{IR spectroscopy}

Both RhPAA and RhAA complexes have an ionised carboxylate group, which indicates that the rhodium interacts with the carboxyl group of the ligand. According to Nakamoto [23], the distance between the antisymmetric and the symmetric vibration of the $\mathrm{COO}^{-}$group (denoted as $\Delta$ ) gives information about the mode of interaction with a metal atom. Unidentate complexes show $\Delta$-values which are much larger than the ionic form, whereas with chelating complexes $\Delta$ is significantly smaller. Finally, the $\Delta$-values of bridging complexes (bridging between two rhodium atoms) are close to the ionic values. Borowski [7] showed that the $\Delta$-value of RhPAA is identical to that of NaPAA (i.e., the ionic form). This would mean that the mode of co-ordination of the carboxylate group to rhodium is bridging. Consequently, the complex should be a dimer, as two Rh atoms are needed for a bridging co-ordination. However, the above-described approach was based on formate and acetate ions. For more complex ligands, such as PAA and AA, the situation might be different. If one considers the absolute $\Delta$-values for $\mathrm{Rh}$ acetates, the mode of co-ordination could well be unidentate in the RhPAA and RhAA complexes.

The interaction of Rh with the amine group of PAA is ascertained by the presence of the $\mathrm{Rh}-\mathrm{N}$ band.
Moreover, the $\mathrm{C}-\mathrm{N}$ stretching vibration shows a shift, which indicates that the environment around this bond has changed. The $\mathrm{N}-\mathrm{H}$ band, however, remains present. Thus, the amine functionality is probably not ionised. As the amount of chlorine is negligible in RhPAA, we can, therefore, conclude that rhodium is in the monovalent oxidation state. Only the charge of the ionised carboxyl group is compensated for by the $\mathrm{Rh}(\mathrm{I})$ metal centre. With RhAA it is more difficult to draw conclusions about the possible interaction of $\mathrm{Rh}$ with the amine group. Because of the rich spectrum of $\mathrm{AA}$, no $\mathrm{Rh}-\mathrm{N}$ band can be discerned. The appearance of the $\mathrm{NH}_{2}$ bending band at $1531 \mathrm{~cm}^{-1}$, however, shows that the environment around this group has changed. The two $\mathrm{N}-\mathrm{H}$ stretching minima seem to disappear, indicating a change as well. Unfortunately, on the basis of only these changes an interaction of $\mathrm{Rh}$ with $\mathrm{N}$ cannot be ascertained unambiguously. For RhAA it is, therefore, also possible that the oxidation state of rhodium is monovalent.

Upon ageing under $\mathrm{N}_{2}$, the $\mathrm{C}=\mathrm{O}$ vibration of the carboxyl group appears in the spectrum of RhPAA. Probably, the rhodium is no longer co-ordinated to the carboxylate group. The position of the $\mathrm{C}=\mathrm{O}$ band is not situated at the same position as with pure PAA, because the $\mathrm{Rh}$ is still present in the environment of the carboxyl group. As no changes are observed in the IR spectra when the RhPAA is exposed to air for a few days, the process must be a slow one and is likely to be initiated by traces of air.

\subsection{Catalytic activity}

The bad reproducibility of the initial activities of the complexes could point out that small rhodium metal particles are formed during the catalytic hydrogenation reaction and that these are at least partially responsible for the observed activities. Therefore, the order with respect to cyclohexene was determined for the RhPAA complexes and for $\mathrm{Rh} / \mathrm{CNFs}$. Due to the variation in initial activities, it was difficult to obtain reliable data for the order measurements. Hence, the obtained orders contain a considerable error and it should be concluded that RhPAA, RhAA and Rh/CNFs have about the same order in cyclohexene. This does not necessarily mean that all the activity of RhPAA and RhAA originates from $\mathrm{Rh}(0)$, but the opposite is not proven either. 
In a separate experiment, solutions of RhPAA and RhAA in DMF were flushed with $\mathrm{H}_{2}$ and the complexes were isolated by evaporation of the DMF. The IR spectra show $\mathrm{C}=\mathrm{O}$ stretching bands at 1643 and $1660 \mathrm{~cm}^{-1}$, respectively. Apparently, some of the complex had disintegrated upon this treatment and rhodium metal had been formed. The formed $\mathrm{Rh}(0)$ could then be responsible for the activity. However, the $\mathrm{C}=\mathrm{O}$ band at $1643 \mathrm{~cm}^{-1}$ was also found with deactivated RhPAA. In that case, the formation of metal particles would lead to a lower activity. The experimental results indicate that the interpretation of the data obtained remains difficult.

If one compares the initial activities of $\mathrm{Rh} / \mathrm{CNFs}$ and deactivated RhAA, taking into account the rhodium content of the catalysts (1\% versus $50 \%$, respectively) and the surface area of the metal particles (diameter $\sim 10$ and $\sim 5 \mathrm{~nm}$ ), the activities compare very well. This means that the activity of this catalyst could well originate from $\mathrm{Rh}(0)$ only. In contrast, the activities of the freshly prepared complexes cannot solely be ascribed to zerovalent rhodium as their activity is too high.

\subsection{Mercury poisoning}

Unfortunately, poisoning with mercury only works in DMF after stirring at $50^{\circ} \mathrm{C}$. This is probably due to the strong co-ordinating properties of DMF. The blank thermal treatment already results in a very strong decrease in activity of RhPAA, which is accompanied by the formation of a $\mathrm{C}=\mathrm{O}$ band at $1643 \mathrm{~cm}^{-1}$. Because of the strong deactivation of the RhPAA complex upon stirring at $50^{\circ} \mathrm{C}$, the mercury poisoning experiment is difficult to interpret. With RhAA, on the other hand, the blank experiment shows an increase in activity, which can be totally ascribed to $\operatorname{Rh}(0)$ as the activity is subsequently blocked by $\mathrm{Hg}$. Clearly, the stability of the complexes is such that stirring at $50^{\circ} \mathrm{C}$ already causes major changes in their chemical state. Therefore, the results must be interpreted with much care.

\subsection{General discussion}

The results presented above have made clear that the two systems under investigation are not simple. However, by careful analysis of the data obtained, some general remarks can be made about the composition of the complexes. Table 4 summarises the essential conclusions. It is likely that both RhPAA and RhAA complexes already contain $\mathrm{Rh}(0)$ after synthesis. This is due to the reducing properties of ethanol. Not all rhodium is, therefore, complexed to PAA. However, all PAA is complexed to Rh, because the carboxyl vibration is completely replaced by those of the carboxylate ion. With PAA the interaction with the metal proceeds via the carboxyl and the amine functionality, whereas with AA only the interaction with the $\mathrm{COO}^{-}$group has been unambiguously established. Based on the elemental analysis data, it is likely that RhAA contains more $\mathrm{Rh}(0)$ than RhPAA. Probably, PAA is a stronger complexing agent than AA. Although the presence of monomeric ligand-metal species is very likely, it is also possible that dimers or oligomers are present. The carboxylate as well as the chloride group could act as a bridge. It is also possible that a mixture of different structures exists. Reduction with $\mathrm{NaBH}_{4}$ does

Table 4

Summary of results after compilation of all data

\begin{tabular}{llll}
\hline Synthesis & Reduction $\mathrm{NaBH}_{4}$ & Catalytic reaction & Storage under $\mathrm{N}_{2}$ \\
\hline RhPAA & & Complex and $\mathrm{Rh}(0)$ is active & Oligomerisation complex or formation \\
PAA complexed to $\mathrm{Rh}(\mathrm{I})$ via & & & big Rh particles \\
$\quad \mathrm{COO}^{-}$and $\mathrm{N}$ & & \\
Formation of $x \mathrm{Rh}(0)$ & & Oligomerisation complex or formation \\
RhA & Same or more $\mathrm{Rh}(0)$ & $\mathrm{Rh}(0)$ is active and probably \\
AA complexed to $\mathrm{Rh}(\mathrm{I})$ via & & & complex as well \\
$\quad \mathrm{COO}^{-}$and may be $\mathrm{N}$ & & & \\
$\begin{array}{l}\text { Formation of } y \mathrm{Rh}(0) \\
y>x\end{array}$ & & &
\end{tabular}


not bring about much changes. May be some more $\mathrm{Rh}(0)$ is formed. As discussed above, it is plausible that rhodium is already in the monovalent oxidation state in RhPAA before reduction with sodium borohydride. Furthermore, no changes in the IR spectrum or in the catalytic activity were observed upon reduction with $\mathrm{NaBH}_{4}$. The catalytic activity observed with RhPAA is partially caused by the complex and partially by $\operatorname{Rh}(0)$. With $\operatorname{RhAA} \mathrm{Rh}(0)$ is surely active and probably the complex as well. During reaction zerovalent rhodium is also formed, which accounts for the bad reproducibility of the activities. Storage under $\mathrm{N}_{2}$ results in a drastic decrease in activity. This could be due to the formation of big Rh particles, initiated by traces of air, that are not very active. However, stirring at $50^{\circ} \mathrm{C}$ results in the formation of $\mathrm{Rh}$ particles with RhAA, which increase the activity considerably. Therefore, it is probably more likely that the structure of the complex itself changes. Oligomerisation of the complex, accompanied by a loss in activity, could be a plausible explanation.

\section{Conclusions}

The IR results have shown that complexes of rhodium with PAA and AA have been formed. With both ligands interaction of $\mathrm{Rh}$ with the carboxyl group is observed. With PAA the Rh is also bonded to the amine group, while this interaction is not certain in RhAA. In view of the synthesis procedure and the characterisation results, it is also likely that $\mathrm{Rh}(0)$ is already present in the as-synthesised complexes. The catalytic activity probably originates from both the metal complexes and small rhodium metal particles. With RhPAA surely part of the activity arises from the complex itself. With RhAA it is likely that the complex itself is active as well, although the results are more difficult to interpret.

The results described above have made clear that the RhPAA and RhAA systems are not pure and that no clear structure of the complexes can be given. The as-selective synthesis procedure is responsible for these observations. Therefore, it can be concluded that the immobilisation of these complexes should proceed by first attaching the ligand and then the metal, because simultaneous immobilisation of ligand and metal would lead to a non-uniform system. Further- more, the catalytic behaviour of the immobilised catalyst should not be compared with its "homogeneous" counterpart, because the measured catalytic activity originates from zerovalent rhodium as well as from the metal-ligand complex.

\section{Acknowledgements}

This work was supported by the Netherlands' Organisation for Scientific Research (NWO).

\section{References}

[1] O.N. Efimov, M.L. Khidekel', V.A. Avilov, P.S. Chekrii, O.N. Eremenko, A.G. Ovcharenko, J. Gen. Chem. USSR (Engl. Trans.) 38 (1968) 2581.

[2] V.A. Avilov, Yu.G. Borod'ko, V.B. Panov, M.L. Khidekel', P.S. Chekrii, Kinet. Catal. (Engl. Trans.) 9 (1968) 582.

[3] O.N. Efimov, O.N. Eremenko, A.G. Ovcharenko, M.L. Khidekel', P.S. Chekrii, Bull. Acad. Sci. USSR, Div. Chem. Sci. (Engl. Trans.) (1969) 778.

[4] V.A. Avilov, M.L. Khidekel', O.N. Eremenko, O.N. Efimov, A.G. Ovcharenko, P.S. Chekrii, US Patent 3,755,194 (1973).

[5] E.L. Muetterties, F.J. Hirsekorn, J. Am. Chem. Soc. 96 (12) (1974) 4063.

[6] N.L. Holy, Fundam. Res. Homogeneous Catal. 3 (1979) 691.

[7] A.F. Borowski, Transition Met. Chem. 8 (1983) 266.

[8] R.P.A. Sneeden, Organochromium Compounds, Academic Press, New York, 1975, p. 114.

[9] I. Rajca, A. Borowski, In: Proceedings of the Symposium of Rhodium Homogeneous Catal., 1978.

[10] I. Rajca, A. Borowski, A. Marzec, Erdöl und Kohle 35 (1) (1982) 36

[11] I.W. Rajca, V.V. Abalayeva, A. Borowski, Fuel 61 (1982) 1292.

[12] N.L. Holy, Tetrahedron Lett. 18 (42) (1977) 3703.

[13] N.L. Holy, Chemtech 10 (1980) 366.

[14] E.N. Frankel, J.P. Friedrich, T.R. Bessler, W.F. Kwolek, N.L. Holy, J. Am. Oil Chem. Soc. 57 (1980) 349.

[15] R.H. Crabtree, The Organometallic Chemistry of the Transition Metals, 2nd Edition, Wiley, New York, 1994, p. 219.

[16] D.R. Anton, R.H. Crabtree, Organometallics 2 (1983) 855.

[17] G.M. Whitesides, M. Hackett, R.L. Brainard, J.-P.P.M. Lavalleye, A.F. Sowinski, A.N. Izumi, S.S. Moore, D.W. Brown, E.M. Staudt, Organometallics 4 (1985) 1819.

[18] Y. Lin, R.G. Finke, Inorg. Chem. 33 (1994) 4891.

[19] K.S. Weddle, J.D. Aiken III, R.G. Finke, J. Am. Chem. Soc. 120 (1998) 5653.

[20] P. Scherrer, Gött. Nachr. 2 (1918) 98.

[21] B.S. Furniss, A.J. Hannaford, P.W.G. Smith, A.R. Tatchell, Vogel's Textbook of Practical Organic Chemistry, 5th Edition, Longman, London, 1989, p. 254. 
[22] V.M. Frolov, L.P. Shuikina, K.K. Turisbekova, G.N. Bondarenko, Kinet. Catal. (Engl. Trans.) 35 (6) (1994) 800.

[23] K. Nakamoto, Infrared and Raman Spectra of Inorganic and Coordination Compounds, B. Applications in Coordination, Organometallic, and Bioinorganic Chemistry, 5th Edition, Wiley, New York, 1997.

[24] G.C. Bond, Heterogeneous Catalysis: Principles and Applications, 2nd Edition, Clarendon Press, Oxford, 1987, p. 49.
[25] M.J. Bennett, P.B. Donaldson, Inorg. Chem. 16 (7) (1977) 1581.

[26] M.C. Baird, D.N. Lawson, J.T. Mague, J.A. Osborn, G. Wilkinson, Chem. Commun. (1966) 129.

[27] M.T. Atlay, L. Carlton, G. Read, J. Mol. Catal. 19 (1983) 57.

[28] C. Larpent, R. Dabard, H. Patin, New J. Chem. 12 (1988) 907.

[29] B. Richter, A.L. Spek, G. van Koten, B.-J. Deelman, J. Am. Chem. Soc. 122 (16) (2000) 3945. 